Rev. Elev. Méd. vét. Pays trop., 1973, 26 (4) : 397-404

\title{
Contribution à l'étude physio-pathologique de la tuberculose bovine
}

\author{
par Y. CHENEAU (*) et J. M. BLANCOU (*) \\ (avec la collaboration technique de Françoise ALEXANDRE, D. RAMBELOMANANA \\ et J, L. POULAIN)
}

\section{RESUME}

L'étude biochimique du sang de bovins atteints de tuberculose révèle d'importantes modifications de sa composition par rapport à celle d'animaux de même race et sains, qui se traduisent par une augmentation du fibrinogène, une augmentation des protéines sériques et par de profonds bouleversements du protéinogramme: baisse des albumines, augmentation des gamma-globulines et abaissement corrélatif du rapport albuminesglobulines.

Ces importantes modifications peuvent permettre de soupçonner les formes les plus graves de la tuberculose bovine.

Dans une précédente étude, différentes techniques pratiques de diagnostic de la tuberculose bovine ont été passées en revue (1). S'il y a été reconnu que la tuberculination intradermique constitue une méthode de choix, mais d'utilisation restreinte à Madagascar, un problème grave subsiste : celui de l'élimination des bovins suspects d'anergie, notamment pour le triage des animaux destinés à des élevages intensifs ou à des établissements d'embouche industrielle.

Car si l'examen sérologique par la technique de double diffusion en gélose permet d'écarter 35 p. 100 des animaux porteurs de lésions organiques graves, un grand nombre d'anergiques restent indétectables par cette méthode.

Or les formes généralisées et contagieuses de la tuberculose ont un retentissement sur la physiologie de l'animal qui se traduit par des modifications des constantes biologiques de l'individu (9). La présente étude a donc pour

(*) I.E.M.V.T., Laboratoire Central de l'Elevage, B.P. 862, Tananarive, République Malgache. objet de déterminer les constantes affectées par la maladie et d'étudier l'ampleur de leurs variations, en se référant à des témoins sains. Les modifications physio-pathologiques étant appréciées, sont-elles significatives de l'état tuberculeux ? Peut-on, grâce à elles, établir un diagnostic de tuberculose généralisée?

\section{MATERIEL UTILISE}

Les analyses ont porté exclusivement sur des zébus malgaches, mâles castrés, âgés de 4 à 8 ans, abattus dans un abattoir industriel de Tananarive.

Les prélèvements, effectués lors de la saignée, ont été recueillis dans des flacons contenant ou non des anticoagulants selon la nature des dosages à réaliser.

Les animaux abattus ont été ensuite autopsiés de façon détaillée (inspection du système ganglionnaire, des viscères et de la carcasse).

L'étude a été conduite en deux étapes : 


\section{Recherches générales}

Une première série de prélèvements portant sur 96 animaux, dont 77 tuberculeux à divers stades d'évolution, et 19 témoins sains, a été soumise à l'analyse de 27 constituants biochimiques, ainsi qu'à la détermination des éléments cytologiques et des résistances globulaires. L'étude statistique de ces résultats préliminaires a permis d'écarter d'emblée de nombreux constituants, dont la variation n'est pas significative.

\section{Recherches complémentaires}

Une seconde série de prélèvements portant sur 210 animaux, dont 80 témoins indemnes de tuberculose, et 130 animaux porteurs de lésions tuberculeuses à divers stades d'évolution, a fait l'objet du dosage de 10 constituants biochimiques, dans le but de confirmer les observations préliminaires.

\section{TECHNIQUES UTILISEES}

Comme les méthodes d'analyses biologiques que nous avons utilisées ont été en tous points semblables à celles mises en œuvre par R. GAULIER à l'occasion de son travail «Etude biochimique, biophysique et cytologique du sang de zébus malgaches (animaux d'abattoir) », qui a été publié dans cette même revue (4) nous ne pouvons mieux faire que d'inviter le lecteur, que ces questions de méthodes pourraient intéresser, à se reporter à cette publication.

\section{RESULTATS}

\section{Recherches générales}

L'ensemble des résultats figure au tableau $n^{n}$ I. Les autopsies des animaux tuberculeux ont permis de les classer en quatre groupes, selon la gravité et l'étendue des lésions observées :

-. groupe A: complexe primaire dissocié ou non;

- groupe B : complexe primaire avec dissémination à un ou plusieurs ganglions non viscéraux;

- groupe C: complexe primaire avec dissémination à un ou plusieurs ganglions viscéraux, organes ou séreuses.
- groupe D: tuberculose généralisée.

Pour chaque type d'analyse sont indiqués par groupe d'animaux la moyenne et l'erreur type. Pour chaque groupe d'animaux malades (A, B, C, D) on donne en outre la valeur de a $t$ » tiré de la comparaison avec les animaux sains et l'indication de la signification statistique.

\section{Recherches complémentaires}

L'étude des lipides totaux, des protéines sériques et des protéinogrammes, du fibrinogène, de la bilirubine et de la teneur en calcium total a été conduite avec les techniques utilisées pour les études préliminaires sur des effectifs importants, notamment en ce qui concerne les sujets sains et les sujets atteints de tuberculose généralisée (80 et 67 sujets).

Les valeurs obtenues sont résumées dans le tableau $\mathrm{n}^{\circ}$ II.

\section{DISCUSSION}

\section{Recherches générales}

Les valeurs obtenues pour les analyses de prélèvements issus d'animaux tuberculeux variant de manière significative au niveau P 0,05 par rapport aux animaux sains sont les suivantes : lipides totaux (groupe D), protéines sériques (groupes $\mathrm{C}$ et $\mathrm{D}$ ) et protéinogramme (groupes A, B, C, D), bilirubine totale (groupes $B$ et D), calcium total (groupe D), magnésium (groupe A), cuivre sérique (groupes $\mathrm{A}$, B, C, D), nombres de globules blancs (groupes $\mathrm{A}, \mathrm{B}, \mathrm{C}$ ) et de polynucléaires neutrophiles (groupe C).

Les résultats obtenus s'écartent parfois de ceux donnés par GAULIER (4). Cela est dû à ce que cet auteur a fait ses dosages sur des animaux tout venant, y compris les malades, alors que nous n'avons pris pour témoins que des animaux cliniquement en bonne santé, n'ayant montré après l'abattage aucune lésion.

\section{Remarque}

Des techniques simples ont été également mises en cuvre sur 180 prélèvements :

- appréciation des gamma-globulines par la méthode néphélémétrique de La Huerga-Popper (7); 
TABLEAU $N^{\circ}$ I

Recherches générales

\begin{tabular}{|c|c|c|c|c|c|}
\hline & Animaux sains & & Animaux $t$ & berculeux & \\
\hline & Groupe $T=19$ & Groupe $A=28$ & Groupe $B=11$ & Groupe $\mathrm{C}=20$ & Groupe $\mathrm{D}=18$ \\
\hline & Va leur moyenne $m \pm \mathrm{Sm}$ & $\frac{\text { Valeur moyenne } m \pm \mathrm{Sm}}{0.37+0.017}$ & Valeur moyenne $\mathrm{m} \pm \mathrm{Sm}$ & Valeur moyenne $\mathrm{m} \pm \mathrm{Sm}$ & $\begin{array}{l}\text { Valeur moyenne } m \pm \text { sm } \\
0,36+0,017\end{array}$ \\
\hline Urêe $\quad(g / 1)$ & $0,34 \pm 0,020$ & $t=0,96$ (N.S.) & $\begin{array}{l}0,41 \pm 0,044 \\
t=1,53 \text { (N.S.) }\end{array}$ & $t=0,13$ (N.S.) & $t=0, \overline{6} 1$ (N.S.) \\
\hline Glucose $(g / 1)$ & $0,82 \pm 0,043$ & $\begin{array}{r}0,74 \pm 0,036 \\
t=1,37 \text { (N.S.) } \\
\end{array}$ & $\begin{array}{l}0,76+0,065 \\
t=0,79 \text { (N.S.) }\end{array}$ & $\begin{array}{r}0,78 \pm 0,033 \\
t=0,80 \text { (N.S.) } \\
\end{array}$ & $\begin{array}{l}0,82 \pm 0,046 \\
t=0,022 \text { (N.S.) }\end{array}$ \\
\hline Cholestérol total $(g / 1)$ & $1,22 \pm 0,053$ & $\begin{array}{r}1,25 \pm 0,043 \\
t=0,42 \text { (N.S.) } \\
\end{array}$ & $\begin{array}{l}1,14 \pm 0,050 \\
t=0,93 \text { (N.S.) }\end{array}$ & $\begin{array}{l}1,16+0,044 \\
t=0,84 \text { (N.S.) }\end{array}$ & $\begin{array}{r}1,09+0,050 \\
t=1,73 \text { (N.S.) }\end{array}$ \\
\hline Lipides totaux $(g / 1)$ & $4,91 \pm 0,343$ & $\begin{array}{r}4,53+0,172 \\
t=1,100 \text { (N.S.) } \\
\end{array}$ & $\begin{array}{r}4,05 \pm 0,372 \\
t=1,61 \text { (N.S.) } \\
\end{array}$ & $\begin{array}{r}4,19 \pm 0,310 \\
t=1,55 \text { (N.S.) }\end{array}$ & $\begin{array}{r}3,62+0,220 \\
t=3,12 \text { (S.) } \\
\end{array}$ \\
\hline Protéines sériques $(\mathrm{g} / 1)$ & $83,52 \pm 1,550$ & $\begin{array}{l}85,10+1,950 \\
t=0,58 \text { (N.S.) }\end{array}$ & $\begin{array}{l}84,90+1,423 \\
\mathrm{t}=0,59 \text { (N.S.) }\end{array}$ & $\begin{array}{l}88,10+1,374 \\
t=2,21 \text { (S.) } \\
\end{array}$ & $\begin{array}{l}92,44 \div 2,564 \\
t=3,01 \text { (S.) }\end{array}$ \\
\hline Fibrinogène $(\mathrm{g} / 1)$ & $8,71 \pm 0,367$ & $\begin{array}{r}7,24 \pm 0,293 \\
t=3,14(\mathrm{~s} .) \\
\end{array}$ & $\begin{array}{r}7,25 \pm 0,592 \\
t=2,21 \text { (S.) } \\
\end{array}$ & $\begin{array}{r}7,84 \pm 0,358 \\
t=1,68 \text { (N.S.) }\end{array}$ & $\begin{array}{l}8,22+0,424 \\
t=0,86 \text { (N.S.) }\end{array}$ \\
\hline Hémoglobine $(\mathrm{g} / 1)$ & $117,84 \pm 2,640$ & $\begin{array}{r}120,14+3,3008 \\
t=0,54 \text { (N.S.) }\end{array}$ & $\begin{array}{r}113,54 \pm 4,466 \\
t=0,88 \text { (N.S.) }\end{array}$ & $\begin{array}{r}115,05+3,956 \\
t=0,58 \text { (N.S.) }\end{array}$ & $\begin{array}{l}114,3 \pm 5,173 \\
t=0,61(\mathrm{~N} . \mathrm{S} .)\end{array}$ \\
\hline Azote "polypeptidique" (mg/1) & $30,26 \pm 3,426$ & $\begin{array}{l}29,82 \pm 2,110 \\
t=0,11 \text { (N.S.) }\end{array}$ & $\begin{array}{l}26,40 \pm 2,447 \\
t=0,78 \text { (N.S.) }\end{array}$ & $\begin{array}{l}31,83 \pm 1,915 \\
t=0,40 \text { (N.S.) }\end{array}$ & $\begin{array}{l}26,43 \pm 1,479 \\
t=1,00 \text { (N.S.) }\end{array}$ \\
\hline Bilirubine totale (mg/l) & $4,04 \pm 0,351$ & $\begin{array}{r}2,39+0,278 \\
\mathrm{t}=3,70(\mathrm{~S} .)\end{array}$ & $\begin{array}{r}3,63 \pm 0,497 \\
\mathrm{t}=0,688 \text { (N.S.) }\end{array}$ & $\begin{array}{r}3,03+0,381 \\
\mathrm{t}=1,94 \text { (N.S.) } \\
\end{array}$ & $\begin{array}{l}2,183 \pm 0,203 \\
t=4,51 \text { (H.S.) }\end{array}$ \\
\hline Créatinine préformée (mg/l) & $2,19 \pm 0,222$ & $\begin{array}{r}2,07 \pm 0,084 \\
\mathrm{t}=0,57 \text { (N.S.) }\end{array}$ & $\begin{array}{l}2,10+0,171 \\
\mathrm{t}=0,26 \text { (N.S.) }\end{array}$ & $\begin{array}{l}2,06+0,072 \\
t=0,59 \text { (N.S.) }\end{array}$ & $\begin{array}{l}2,00 \pm 0,180 \\
\mathrm{t}=0,67 \text { (N.S.) }\end{array}$ \\
\hline Chlore globulaire $(\mathrm{g} / 1)$ & $2,03 \pm 0,065$ & $\begin{array}{l}2,07 \pm 0,052 \\
t=0,05 \text { (N.S.) }\end{array}$ & $\begin{array}{l}1,93 \pm 0,055 \\
t=1,03 \text { (N.S.) }\end{array}$ & $\begin{array}{l}1,96 \pm 0,056 \\
t=0,73 \text { (N.S.) }\end{array}$ & $\begin{array}{l}1,96 \pm 0,042 \\
t=0,83 \text { (N.S.) }\end{array}$ \\
\hline Chlore plasmatique $(\mathrm{g} / \mathrm{l})$ & $3,19 \pm 0,059$ & $\begin{array}{r}3,31 \pm 0,062 \\
t=1,40(\mathrm{~N} . \mathrm{S} .)\end{array}$ & $\begin{array}{r}3,17 \pm 0,047 \\
t=0,26 \text { (N.S.) }\end{array}$ & $\begin{array}{l}3,23 \pm 0,047 \\
t=0,60 \text { (N.S.) }\end{array}$ & $\begin{array}{l}3,18 \pm 0,068 \\
t=0,07 \text { (N.S.) }\end{array}$ \\
\hline $\begin{array}{l}\text { Rapport érythroplasmatique } \\
\text { chlore }\end{array}$ & $0,63 \pm 0,014$ & $\begin{array}{r}0,62+0,012 \\
t=0,70 \quad(\text { N.S. }) \\
\end{array}$ & $\begin{array}{r}0,61 \pm 0,020 \\
t=1,52 \text { (N.S.) }\end{array}$ & $\begin{array}{r}0,60 \div 0,028 \\
t=0,96 \text { (N.S.) } \\
\end{array}$ & $\begin{array}{l}0,61 \pm 0,010 \\
\mathrm{t}=1,75 \text { (N.S.) }\end{array}$ \\
\hline Phosphore minéral (mg/1) & $70,10 \pm 3,951$ & $\begin{array}{l}85,0= \pm 5,409 \\
t=2,02 \text { (N.S.) }\end{array}$ & $\begin{array}{l}75,36 \pm 4,334 \\
t=0,85 \text { (N.S.) }\end{array}$ & $\begin{array}{l}68,35 \pm 2,673 \\
t=0,37 \text { (N.S.) }\end{array}$ & $\begin{array}{l}73,66 \pm 3,675 \\
t=0,65 \text { (N.S.) }\end{array}$ \\
\hline Calcium total (mg/1) & $89,68 \pm 1,827$ & $\begin{array}{l}84,53 \pm 2,564 \\
t=1,48 \text { (N.S.) }\end{array}$ & $\begin{array}{l}84,63 \pm 2,111 \\
t=1,74 \text { (N.S.) }\end{array}$ & $\begin{array}{l}87,65 \pm 1,599 \\
t=0,84(\mathrm{~N}, \mathrm{~S},)\end{array}$ & $\begin{array}{c}80,77 \pm 1,342 \\
t=3,89 \text { (s.) }\end{array}$ \\
\hline Magnésium (mg/1) & $21,63 \pm 1,351$ & $\begin{array}{l}27,51+1,823 \\
t=2,39(\mathrm{~S} .)\end{array}$ & $\begin{array}{l}22,36 \pm 2,842 \\
t=0,26 \text { (N.S.) }\end{array}$ & $\begin{array}{l}25,45 \pm 1,884 \\
t=1,63 \text { (N.S.) }\end{array}$ & $\begin{array}{l}25,11 \pm 1,926 \\
t=1,49 \text { (N.S.) }\end{array}$ \\
\hline Sodium $(g / 1)$ & $3,31 \pm 0,030$ & $\begin{array}{l}3,38 \pm 0,042 \\
t=1,23 \text { (N.S.) }\end{array}$ & $\begin{array}{r}3,30 \pm 0,022 \\
t=0,07 \text { (N.S.) } \\
\end{array}$ & $\begin{array}{r}3,33+0,040 \\
\mathrm{E}=0,43 \text { (N.S.) } \\
\end{array}$ & $\begin{array}{l}3,34 \pm 0,047 \\
\mathrm{t}=0,53 \text { (N.s.) }\end{array}$ \\
\hline Potassium (mg/1) & $210 \pm 6,992$ & $\begin{array}{l}223,1 \pm 7,245 \\
t=1,25 \text { (N.S.) }\end{array}$ & $\begin{array}{l}208,3 \pm 30,720 \\
t=0,06 \text { (N.S.) }\end{array}$ & $\begin{array}{l}198,5+4,636 \\
t=1,38 \text { (N.S.) }\end{array}$ & $\begin{array}{r}221,1 \pm 6,758 \\
t=1,14 \text { (N.S.) }\end{array}$ \\
\hline Cuivre sérique ( $(\gamma \mathrm{p} .100 \mathrm{ml})$ & $52,57+3,165$ & $\begin{array}{l}72,4 \pm \pm 6,812 \\
t=2,28(S .)\end{array}$ & $\begin{array}{l}72,3 \pm 10,309 \\
t=2,30(\mathrm{~S} .)\end{array}$ & $\begin{array}{l}82,6 \pm 9,353 \\
t=2,97(\mathrm{~S} .)\end{array}$ & $\begin{array}{r}100,7 \pm 11,042 \\
t=4,52 \text { (H.S.) }\end{array}$ \\
\hline
\end{tabular}


TABLEAU $\mathbb{N}^{\circ}$ I (suite)

Recherches générales

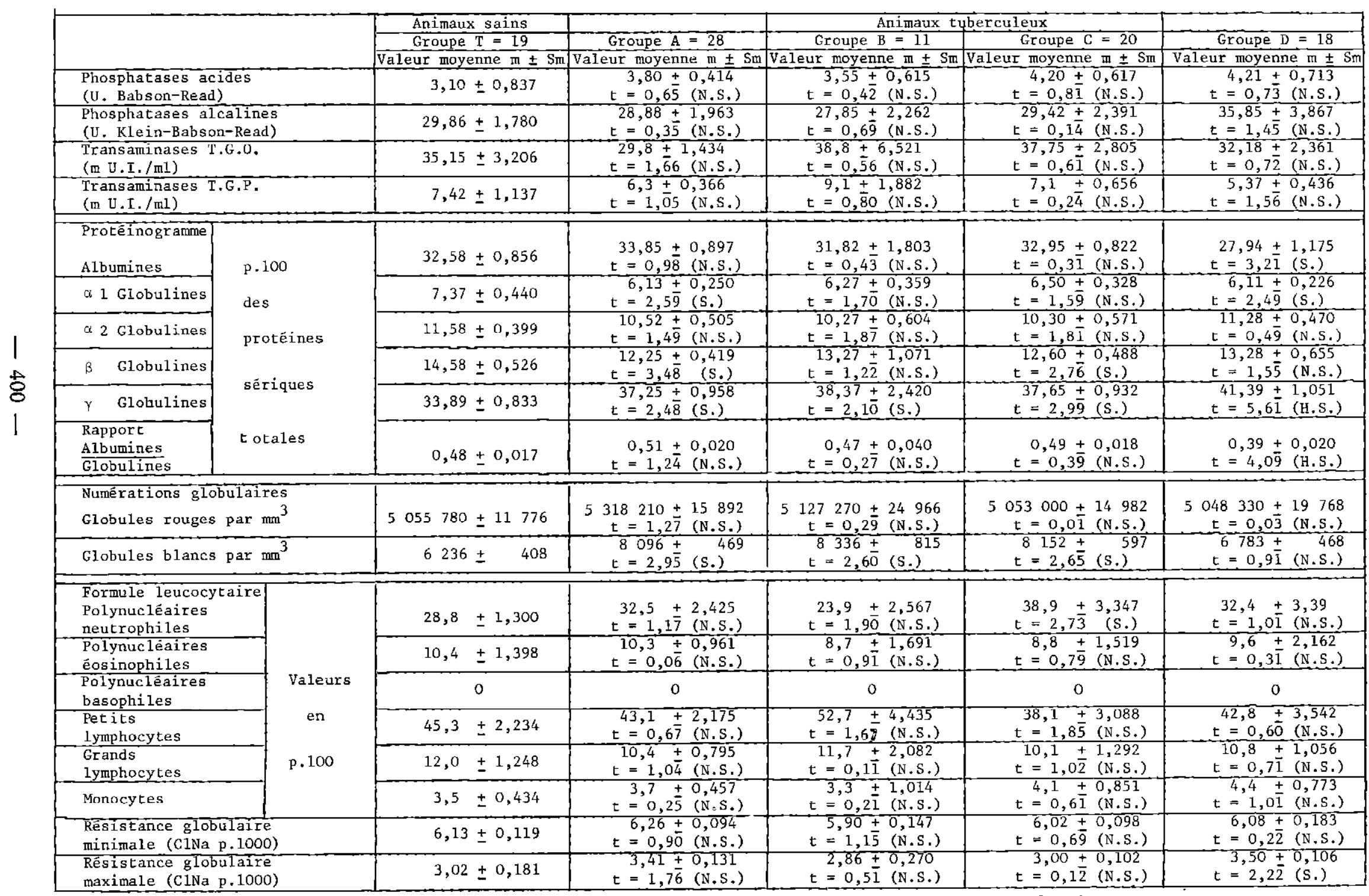


TABLEAU $N^{\circ}$ II

Recherches complémentaires

\begin{tabular}{|c|c|c|c|c|c|}
\hline & Animaux sains & \multicolumn{4}{|c|}{ Animaux tuberculeux } \\
\hline & Groupe $T=80$ & Groupe $A=28$ & Groupe $B=14$ & Groupe $C=21$ & Groupe $D=67$ \\
\hline & Valeur moyenne $\mathrm{m} \pm \mathrm{Sm}$ & Valeur moyenne $\mathrm{m} \pm \mathrm{Sm}$ & Valeur moyenne $m \pm S m$ & Valeur moyenne $m \pm S m$ & Valeur moyenne $\mathrm{m} \pm \mathrm{Sm}$ \\
\hline Lipides totaux & $4,03 \pm 0,129$ & $\begin{array}{c}4,44 \pm 0,180 \\
\mathrm{t}=1,69 \text { (N.S.) }\end{array}$ & $\begin{array}{c}4,35 \pm 0,329 \\
t=0,94 \text { (N.s.) }\end{array}$ & $\begin{array}{c}4,18 \pm 0,198 \\
t=0,5 \frac{1}{6} \text { (N.s.) }\end{array}$ & $\begin{array}{l}3,93+0,154 \\
\mathrm{t}=0,5 \frac{1}{2} \text { (N.S.) }\end{array}$ \\
\hline Protêines sēriques & $85,96 \pm 1,416$ & $\begin{array}{c}90,25 \pm 1,874 \\
t=1,62 \text { (N.S.) }\end{array}$ & $\begin{array}{l}91,14 \pm 2,882 \\
t=1,43 \text { (N.S.) }\end{array}$ & $\begin{array}{c}85,28+1,505 \\
t=0,2 \frac{1}{3} \text { (N.S.) }\end{array}$ & $\begin{array}{c}96,67 \pm 1,439 \\
t=5,26 \text { (H.S.) }\end{array}$ \\
\hline Fibrinogène & $7,67 \pm 0,269$ & $\begin{array}{c}8,15 \pm 0,313 \\
t=0,97 \text { (N.S.) }\end{array}$ & $\begin{array}{c}7,48 \pm 0,250 \\
t=0,29 \text { (N.S.) }\end{array}$ & $\begin{array}{l}\quad 7,73 \pm \stackrel{0,412}{t}=0,1 \overline{1}(\text { N.S. })\end{array}$ & $\begin{array}{r}9,04 \pm 0,330 \\
\left.t=3,2 \frac{4}{4} .\right)\end{array}$ \\
\hline Bilirubine & $6,37 \pm 0,260$ & $\begin{aligned} & 5,75 \pm 0,456 \\
t= & 1,21 \text { (N.S.) }\end{aligned}$ & $\begin{array}{c}4,81 \pm 0,49 \\
\mathrm{t}=2,38 \quad \text { (S.) }\end{array}$ & $\begin{aligned} & 5,82 \pm 0,390 \\
t & =1,00(\mathrm{~N} . \mathrm{S} .)\end{aligned}$ & $\begin{aligned} & 6,82 \pm 0,281 \\
t & =1,17 \text { (N.S.) }\end{aligned}$ \\
\hline Calcium total $(\mathrm{n}=50)$ & $87,18 \pm 1,254$ & - & - & - & $\begin{array}{l}84,18+1,332 \\
\mathrm{t}=1,6 \overline{3} \text { (N.S.) }\end{array}$ \\
\hline Albumines & $32,6 \pm 0,363$ & $\begin{array}{l}31,29 \pm 0,627 \\
t=1,8 \overline{3} \text { (N.S.) }\end{array}$ & $\begin{array}{c}32,21 \pm 0,978 \\
t=0,40 \text { (N.S.) }\end{array}$ & $\begin{array}{l}33,00 \pm 0,759 \\
t=0,49 \text { (N.S.) }\end{array}$ & $\begin{array}{l}25,64 \pm 0,541 \\
t=10,95 \text { (H.S.) }\end{array}$ \\
\hline$\alpha 1$ Globulines & $7,4 \pm 0,184$ & $\begin{array}{r}8,25 \pm 0,358 \\
t=2,32 \quad \text { (S.) }\end{array}$ & $\begin{array}{c}7,29 \pm 0,321 \\
t=0,19 \text { (N.S.) }\end{array}$ & $\begin{array}{c}7,86 \pm 0,302 \\
\mathrm{t}=1,2 \overline{3} \text { (N.S.) }\end{array}$ & $\begin{array}{c}\quad 7,09 \pm 0,205 \\
\mathrm{t}=1,0 \frac{0}{3} \text { (N.S.) }\end{array}$ \\
\hline a 2 Globulines & $10,2 \pm 0,249$ & $\begin{array}{c}10,64 \pm 0,415 \\
t=0,95 \text { (N.S.) }\end{array}$ & $\begin{array}{l}11,0+0,392 \\
t=1,33 \text { (N.S.) }\end{array}$ & $\begin{array}{c}10,90 \pm 0,529 \\
t=1,31 \text { (N.S.) }\end{array}$ & $\begin{array}{l}10,22 \pm 0,281 \\
t=0,13(\text { N.S. })\end{array}$ \\
\hline B Globulines & $12,9 \pm 0,218$ & $\begin{array}{l}13,36 \pm 0,501 \\
t=0,8 \overline{3} \text { (N.S.) }\end{array}$ & $\begin{array}{l}13,50 \pm 0,796 \\
t=0,87 \text { (N.s.) }\end{array}$ & $\begin{array}{l}13,57 \pm 0,45 \\
t=1,25 \text { (N.S.) }\end{array}$ & $\begin{array}{l}13,45 \pm 0,323 \\
t=1,27 \text { (N.S.) }\end{array}$ \\
\hline$\gamma \quad$ Globulines & $36,9 \pm 0,451$ & $\begin{array}{c}36,46 \pm 0,677 \\
t=0,4 \overline{9} \text { (N.S.) }\end{array}$ & $\begin{array}{l}36,0 \\
t=0,7 \frac{1}{3} \text { (N.S.) }\end{array}$ & $\begin{array}{r}34,67 \pm 0,670 \\
t=2,34 \quad(s .)\end{array}$ & $\begin{array}{l}43,60 \pm 0,632 \\
t=8,82 \text { (H.S.) }\end{array}$ \\
\hline Rapport $: \frac{\text { albumines }}{\text { globulines }}$ & $0,488 \pm 0,008$ & $\begin{array}{c}0,45 \pm 0,013 \\
t=1,8 \overline{8} \text { (N.S.) }\end{array}$ & $\begin{array}{c}0,47 \pm 0,022 \\
\mathrm{t}=0,6 \overline{7} \text { (N.S.) }\end{array}$ & $\begin{array}{c}0,49 \pm 0,014 \\
t=0,4 \frac{1}{6}(\mathrm{~N} . \mathrm{S} .)\end{array}$ & $\begin{array}{c}0,35 \pm 0,010 \\
t=10,80 \text { (H.S.) }\end{array}$ \\
\hline
\end{tabular}


- détermination du delta cryoscopique, de la densité du sang et de la vitesse de sédimentation.

Ces techniques, réalisées dans le but de rendre un éventuel diagnostic plus pratique, ne se sont pas révélées assez sensibles pour permettre de dégager des différences statistiquement significatives.

De même, la protéine anti-C (ou CRP), décrite comme étant le reflet des processus inflammatoires (7), a été recherchée sur 100 prélèvements issus d'animaux tuberculeux : aucun résultat positif n'a été enregistré.

\section{Recherches complémentaires}

A la lecture du tableau $\mathrm{n}^{\circ}$ II une constatation s'impose: seules les tuberculoses généralisées, le plus souvent contagieuses (tuberculoses ouvertes) et qui sont susceptibles de rendre le sujet anergique entraînent des variations importantes de certains constituants biochimiques. Des différences hautement significatives au plan statistique sont observées.

Les variations du taux des lipides totaux, de la bilirubine et du calcium total qui étaient significatives dans les études préliminaires portant sur des effectifs réduits, n'apparaissent plus significatives.

Le taux du fibrinogène est très élevé chez les animaux atteints de tuberculose généralisée, comme il augmente dans un grand nombre d'infections. Un inconvénient : le dosage, réalisé sur plasma, nécessite de recueillir le sang sur mélange de Wintrobe, alors que toutes les autres analyses retenues sont réalisées à partir du sérum.

Les protéines sériques et le protéinogramme montrent les plus grands bouleversements, et l'ampleur des variations est remarquable et hautement significative sur le plan statistique. Le taux des protéines sériques passe de 86 à 97 et $\mathrm{t}=5,26$. Quant au protéinogramme, si les taux d'alpha 1, alpha 2 et bêta globulines ne sont pas modifiés, les taux d'albumines et de gamma-globulines sont très perturbés: chute des albumines, (de 32,6 à 25,6 p. $100 ; \mathrm{t}=$ 10,95), augmentation des gamma-globulines, (de 36,9 à 43,6 p. $100 ; \mathrm{t}=8,82$ ) et corrélativement, baisse du rapport albumines/globulines (de 0,48 à 0,$35 ; t=10,80$ ). Ces modifications sont communes à de nombreux processus inflammatoires.

\section{CONCLUSIONS}

L'analyse comparative des constituants biochimiques de 306 zébus malgaches, atteints de tuberculose ou sains, fait apparaître que les seules différences significatives entre les deux catégories concernent :

- l'augmentation des taux du fibrinogène;

- l'augmentation des protides totaux;

- l'augmentation des gamma-globulines;

- la diminution des albumines.

Ces résultats, non conformes à ceux de BOCHDALEK (2), SINGH et PRASAD (10) et VRZGULA (12), confirment au contraire ceux plus nombreux de BOCHDALEK et TOMAZEWSKA (3), GREVE (5), ILIEV et Collab. (6), VICARD (11), WRIGHT (13).

Aucune de ces modifications ne présente de caractère de spécificité. On les retrouve à des degrés divers dans tous les états inflammatoires importants dus la plupart du temps à des infections, mais aussi à des parasitoses.

Cependant l'on se trouve à Madagascar souvent confronté au problème du dépistage des animaux tubcrculeux anergiques à la tuberculine.

En l'absence de toute autre maladie infectieuse cliniquement décelable et de toute parasitose intense que la coproscopie révélerait (la fasciolose par exemple), nous avons recherché quel parti l'on peut tirer de l'examen du rapport albumines/globulines et de sa combinaison au taux de protéines sériques par la formule linéaire sujvante :

$$
\mathrm{Z}=0,68 x-0,73 \gamma\left\{\begin{array}{l}
\chi=\text { taux de } \\
\text { protéines sériques. } \\
\gamma=\text { rapport } \\
\text { albumines/globul. }
\end{array}\right.
$$

En considérant comme tuberculeux les animaux ayant un rapport albumines/globulines inférieur à 0,41 , nous avons commis 5,4 p. 100 d'erreurs par excès et 12,4 p. 100 d'erreurs par défaut.

En associant protéines sériques et rapport albumines/globulines et en considérant comme tuberculeux les animaux pour lesquels $\mathrm{Z}$ est supérieur à 33, nous avons commis 3,2 p. 100 d'erreurs par excès et 14,6 p. 100 d'erreurs par défaut. 
Dans les deux cas, les erreurs par défaut sont beaucoup plus importantes que les erreurs par excès.

Bien qu'il ne s'agisse pas de moyens faciles à mettre en ouvre, puisqu'ils réclament des dosages de laboratoire, l'examen du rapport albumines/globulines et celui des protéines sériques peuvent, lorsqu'on a éliminé toute autre cause de perturbation, permettre d'apporter un élément au dépistage des tuberculoses évolutives avec anergie tuberculinique (8),

\section{SUMMARY}

\section{Contribution to the physio-pathological study of bovine tuberculosis}

The biochemical study of the blood of cattle infected with tuberculosis reveals some important modification of its composition with regard to the one of healthy and same breed animals, which finds expression in: an increase of fibrinogen, an increase of total proteins rate, and some deeps perturbation of proteinogram: a fall of albumins, an increase of gamma globulins, and a correlative falling off of albumins/globulins ratio.

These important modifications may allowed to found suspicion of most serious forms of bovine tuberculosis.

\section{RESUMEN}

\section{Contribución al estudio fisio-patologico de la tuberculosis bovina}

El estudo bioquimico de la sangre de bovinos atacados por la tuberculosis revela importantes modificaciones de su composición con relación a la de animales de misma raza y sanos que se traducen por: un aumento de fibrinógeno, un aumento de las proteinas sericas, y trastornos hondos del proteinograma: baja de las albúminas, aumento de las gamma globulinas y rebaja correlativa de la relación albuminasglobulinas.

Estas importantes modificaciones pueden hacer sospechar las formas más graves de la tuberculosis bovina.

\section{BIBLIOGRAPHIE}

1. BLANCOU (J. M.). Comparaison de techniques pratiques de diagnostic de la tuberculose bovine. Rev. Elev. Méd. vét. Pays trop., 1972, 25 (1): 29-35.

2. BOCHDALEK (R.). Concentrations of $\mathrm{Na}, \mathrm{K}$, $\mathrm{Ca}$ and inorganic $\mathrm{P}$ in the serum of cattle giving negative and positive reactions to the tuberculin tests. Medycyna Weterynaryjina, 1971, 27 (9): $522-523$.

3. BOCHDALEK (R.), TOMAZEWSKA (B.). Levels of haemagglutinins, total proteins and protein fractions in the serum of tuberculin positive cows after injection of tuberculin. Medycyna Weterynarydiina, 1959, 25 : 345-348.

4. GAULIER (R.). Etude biochimique, biophysique et cytologique du sang de zébus malgaches (animaux d'abattoir). Rev. Elev. Méd. vét. Pays trop., 1970, 23 (4) : 469-477.

5. GREVE (D.). Micro-electrophoretic examination of the blood proteins of cattle in leucosis, brucellosis and T.B. Dtsch. tierarzfl. Wschr., 1955, 62: $536-538$ and 540 .

6. ILIEV (T.), KOICHEV (K.), GIRGINOV (G.). Tuberculosis in cattle: relationships between serum protein pattern, tuberculosis reactions and lesions. Nauch. Trud. Vish. Vet. Med. Inst., Sofia, 1961, $9: 271-278$.
7. LECOQ (R.). Manuel d'analyses médicales et de biologie chimique, 2e ćd., Paris, Doin, 1967.

8. LUCAS (A.), GAYOT (G.). Pathologie de la production du lait: procédés actuels de dépistage de la tuberculose bovine. Ann. Nutr. Alim, 1967, 21 (1) : 1 A - 63 A.

9. Rapports annuels du Laboratoire Central de l'Elevage à Madagascar, Tananarive, archives I.E.M.V.T.

10. SINGH (C. D. N.), PRASAD (J. N.). Some studies on tuberculosis in animals. 1. On the blood picture of positive tuberculin reactors and incidence of tuberculosis in cattle. Indian J. anim. Sci., 1971, 41 (7) : 569-572.

11. VICARD (A.). Contribution à une prophylaxie de la tuberculose bovine. Paris, Vigot éditeurs, 1955.

12. VRZGULA (L.). Serum mineral values in tuberculosis cattle. Vet. Caus., 1961, 10: 149-159.

13. WRIGHT (G. L.), Jr. Electrophoretic and immunoelectrophoretic analyses of serum from normal cows and cows experimentally and naturally infected by mycobacteria. Diss. Abstr., 1967, 27 B, 2240 . 